Note

\title{
ON DOUBLE-STAR DECOMPOSITION OF GRAPHS
}

\author{
Saieed Akbari ${ }^{a}$, Shahab $\mathrm{Haghi}^{b}$ \\ HAMidreZa Maimani $^{b}$ AND ABbas SEIFy ${ }^{1, b}$ \\ ${ }^{a}$ Department of Mathematical Sciences \\ Sharif University of Technology \\ Tehran, Iran, P.O. Box 11365-11155 \\ ${ }^{b}$ Mathematics Section, Department of Basic Sciences \\ Shahid Rajaee Teacher Training University \\ Tehran, Iran, P.O. Box 16783-163 \\ e-mail: s_akbari@sharif.edu \\ sh.haghi@ipm.ir \\ maimani@ipm.ir \\ abbas.seify@gmail.com
}

\begin{abstract}
A tree containing exactly two non-pendant vertices is called a doublestar. A double-star with degree sequence $\left(k_{1}+1, k_{2}+1,1, \ldots, 1\right)$ is denoted by $S_{k_{1}, k_{2}}$. We study the edge-decomposition of graphs into double-stars. It was proved that every double-star of size $k$ decomposes every $2 k$-regular graph. In this paper, we extend this result by showing that every graph in which every vertex has degree $2 k+1$ or $2 k+2$ and containing a 2 -factor is decomposed into $S_{k_{1}, k_{2}}$ and $S_{k_{1}-1, k_{2}}$, for all positive integers $k_{1}$ and $k_{2}$ such that $k_{1}+k_{2}=k$.
\end{abstract}

Keywords: graph decomposition, double-stars, bipartite graph.

2010 Mathematics Subject Classification: 05C51, 05 C05.

\section{REFERENCES}

[1] J. Akiyama and M. Kano, Factors and Factorizations of Graphs (London, Springer, 2011). doi:10.1007/978-3-642-21919-1

[2] A. Bondy and U.S.R. Murty, Graph Theory (Graduate Texts in Mathematics, Springer, 2008).

\footnotetext{
${ }^{1}$ Corresponding author.
} 
[3] S.I. El-Zanati, M. Ermete, J. Hasty, M.J. Plantholt and S. Tipnis, On decomposing regular graphs into isomorphic double-stars, Discuss. Math. Graph Theory 35 (2015) 73-79. doi:10.7151/dmgt.1779

[4] M. Jacobson, M. Truszczyński and Zs. Tuza, Decompositions of regular bipartite graphs, Discrete Math. 89 (1991) 17-27. doi:10.1016/0012-365X(91)90396-J

[5] F. Jaeger, C. Payan and M. Kouider, Partition of odd regular graphs into bistars, Discrete Math. 46 (1983) 93-94.

doi:10.1016/0012-365X(83)90275-3

[6] A. Kötzig, Problem 1, in: Problem session, Proceedings of the Tenth Southeastern Conference on Combinatorics, Graph Theory and Computing, Congr. Numer. XXIV (1979) 913-915.

[7] G. Ringel, Problem 25, in: Theory of Graphs and its Applications, Proc. Symposium Smolenice 1963 (Prague, 1964), 162.

Received 4 August 2015

Revised 9 May 2016

Accepted 9 May 2016 